\title{
Aplicação de um Modelo Híbrido para Predição do Tempo de Vida de Baterias de Li-Po considerando Correntes de Descarga Contantes e Pulsadas
}

\author{
Luana F. Meireles ${ }^{1}$ \\ Rita S. Kusiak ${ }^{2}$ \\ Airam T. Z. R. Sausen ${ }^{3}$ \\ Paulo S. Sausen ${ }^{4}$ \\ Programa de Pós Graduação Stricto Sensu em Modelagem Matemática, UNIJUí, Ijuí, RS
}

\begin{abstract}
Resumo. Neste trabalho é realizada a modelagem matemática do tempo de vida de baterias de Lítio Íon Polímero (Li-Po) utilizando correntes de descargas constantes e pulsadas a partir de um modelo híbrido. Este modelo é formado da união do Modelo Elétrico para Predizer Runtime e Características V-I de uma bateria com o Modelo Cinético de Bateria (KiBaM). A validação do modelo consiste em uma análise comparativa entre os resultados simulados pelo modelo e os resultados experimentais. As simulações computacionais são realizadas na ferramenta computacional Matlab/Simulink e os dados experimentais são obtidos com o auxílio de uma plataforma de testes. A análise realizada mostra que este modelo híbrido apresenta resultados satisfatórios.
\end{abstract}

Palavras-chave. Modelo Híbrido, Baterias, Tempo de Vida

\section{Introdução}

Os dispositivos móveis, tais como, celulares e notebooks tornaram-se indispensáveis em atividades diárias devido a mobilidade e a praticidade apresentada pelos mesmos. A mobilidade é possível a partir da utilização de uma fonte de energia finita, conhecida como bateria. No entanto, o tempo de vida limitado das mesmas tornou-se um problema devido ao aumento do consumo de energia pelas mais diversas aplicações que são executadas nestes dispositivos. Pensando nisto, e buscando baterias que consigam atender a demanda atual, faz-se necessário aprimorar os métodos para a predição do seu tempo de vida. A literatura técnica propõe diversas maneiras de realizar esta predição, entre elas encontra-se a modelagem matemática.

Ao longo dos anos, diferentes modelos matemáticos foram desenvolvidos, dentre estes destacam-se: os modelos eletroquímicos [3], os modelos elétricos [1], os modelos estocásticos [2] e os modelos analíticos $[7,8]$. No entanto, os modelos de baterias existentes

\footnotetext{
${ }^{1}$ luh.fransozi@hotmail.com

${ }^{2}$ ritinha.kusiak@yahoo.com.br

3 airam@unijui.edu.br

${ }^{4}$ sausen@unijui.edu.br
} 
possuem pelo menos uma desvantagem e, com o intuito de solucionar esta desvatagem surgem os modelos híbridos [5,10], que possibilitam a união de dois ou mais modelos existentes e com características diferentes. Assim, é possível obter um novo modelo que possui as vantagens de ambos.

Neste contexto, o principal objetivo deste trabalho é realizar a modelagem matemática do tempo de vida de baterias de Lítio Íon Polímero (Li-Po) utilizando um modelo híbrido. Para isto, é realizado o estudo e aplicação de um modelo híbrido, desenvolvido em [5], capaz de capturar as características elétricas e o comportamento não linear da bateria para diferentes perfis de correntes de descarga constantes e variadas. Este modelo é concebido a partir da união do modelo elétrico para Predizer Runtime e Características V-I de uma bateria com o modelo KiBaM. O modelo é implementado na ferramenta computacional MatLab/Simulink, e os resultados das simulações, são comparados com dados experimentais de uma plataforma de testes, utilizando baterias Li-Po, modelo PL 383562-2C. Destaca-se que a validação é realizada para correntes de descargas constantes e variadas.

O restante deste capítulo está organizado como segue. Na Seção 2 são descritas as equações do modelo. Na Seção 3 são apresentados os resultados e discussões, ou seja, a obtenção dos dados experimentais, a estimação dos parâmetros do modelo e a validação do modelo. Por fim, na Seção 4 são apresentadas as conclusões.

\section{Modelo Híbrido de Baterias}

Nesta seção é apresentado o modelo híbrido estudado neste trabalho, que é obtido através da conexão do modelo elétrico para Predizer Runtime e Características V-I de uma bateria com o modelo analítico KiBaM.

O modelo elétrico descreve com precisão as características do circuito dinâmico da bateria, entretanto, utiliza um capacitor constante para modelar a capacidade da mesma, desconsiderando os efeitos não lineares $[7,8]$. Buscando agregar ao modelo estes efeitos, substituiu-se os componentes responsáveis pelo estado de carga e o tempo de vida da bateria, no modelo elétrico, por equações baseadas no modelo KiBaM. Esta alteração possibilita incorporar ao modelo elétrico o efeito de recuperação e o efeito da taxa de capacidade, presentes no modelo analítico.

O modelo híbrido considera um período total de $t_{0}<t<t_{r}$, em que no período de $t_{0}<t<t_{d}\left(\operatorname{com} t_{d}<t_{r}\right)$ a bateria é descarregada com uma corrente constante $i=I>0$, e então repousa no restante do período, ou seja, $t_{d}<t<t_{r}$, com $i=0$. O estado de carga $(S O C)$ é descrito por

$$
S O C(t)=\frac{C_{a}(t)}{C_{m}}
$$

onde: $C_{a}(t)$ é a capacidade disponível da bateria e $C_{m}$ é a capacidade nominal da bateria.

A capacidade disponível da bateria, $C_{a}(t)$, é determinada por

$$
C_{a}(t)=C_{i}-l(t)-C_{u}(t),
$$

onde: $C_{i}$ é a capacidade inicial da bateria, $l(t)$ é a carga total consumida pelo sistema dada por 


$$
l(t)=\int i(t) d t
$$

e a $C_{u}(t)$ representa a capacidade indisponível no tempo $t$ e é descrita pela carga indisponível proveniente do modelo KiBaM

$$
C_{u}(t)=\left\{\begin{array}{c}
C_{u}\left(t_{0}\right) \cdot e^{-k^{\prime}\left(t-t_{0}\right)}+(1-c) \cdot \frac{I}{c} \cdot\left(\frac{1-e^{-k^{\prime}\left(t-t_{0}\right)}}{k^{\prime}}\right), t_{0}<t<t_{d} \\
C_{u}\left(t_{d}\right) \cdot e^{-k^{\prime}\left(t-t_{d}\right)}, t_{d}<t<t_{r},
\end{array}\right.
$$

onde: $C_{u}\left(t_{0}\right)$ é a capacidade indisponível da bateria no início da descarga, $c$ é uma fração da capacidade total $(C)$ da bateria, $C_{u}\left(t_{d}\right)$ é a capacidade indisponível da bateria no final do tempo de descarga, $t_{0}$ é o tempo inicial, $t_{d}$ é o tempo final da descarga, $t_{r}$ é o tempo que resta para terminar o período, $k^{\prime}$ é uma constante relacionada com a taxa de difusão de energia entre as fontes e $I$ é a corrente de descarga.

Assim, o estado de carga $(S O C)$ é dado por

$$
S O C(t)=S O C_{i}-\frac{1}{C_{m}} \cdot\left[\int i(t) d t+C_{u}(t)\right]
$$

onde: $S O C_{i}$ é o estado de carga inicial.

A tensão do modelo é proveniente do modelo elétrico e é representada por

$$
V(t)=V_{o c}[S O C(t)]-i(t) \cdot R_{s}[S O C(t)]-V_{t}(t)
$$

onde: $V(t)$ é a tensão, $V o c[S O C(t)]$ é a tensão de circuito aberto, $R_{s}[S O C(t)]$ é a resistência em série e $V_{t}(t)$ é a tensão transiente. Os elementos da equação (6) são determinados pelas equações

$$
\begin{gathered}
V_{o c}[S O C(t)]=a_{0} \cdot e^{a_{1}[S O C(t)]}+a_{2}+a_{3}[S O C(t)]-a_{4}[S O C(t)]^{2}+a_{5}[S O C(t)]^{3} \\
R_{s}[S O C(t)]=b_{0} \cdot e^{-b 1[S O C(t)]}+b_{2}+b_{3}[S O C(t)]-b_{4}[S O C(t)]^{2}+b_{5}[S O C(t)]^{3} \\
V_{t}(t)=V_{t S}(t)+V_{t L}(t)
\end{gathered}
$$

onde: $V_{t S}(t)$ é a tensão transiente de curta duração dada pela equação (10) e $V_{t L}(t)$ é a tensão transiente de longa duração dada pela equação (11)

$$
V_{t S}(t)=\left\{\begin{array}{c}
R_{t S} \cdot i(t) \cdot\left[1-e^{-\frac{\left(t-t_{0}\right)}{\tau_{S}}}\right], \quad t_{0}<t<t_{d} \\
V_{t S}\left(t_{d}\right) \cdot e^{-\frac{\left(t-t_{d}\right)}{\tau_{S}}}, \quad t_{d}<t<t_{r}
\end{array}\right.
$$

onde: $R_{t S}$ é a resistência transiente de curta duração, $V_{t S}\left(t_{d}\right)$ é a tensão transiente de curta duração no tempo final de descarga, $\tau_{S}=R_{t S} \cdot C_{t S}$ e $C_{t S}$ é a capacitância transiente de curta duração.

$$
V_{t L}(t)=\left\{\begin{array}{c}
R_{t L} \cdot i(t) \cdot\left[1-e^{-\frac{\left(t-t_{0}\right)}{\tau_{L}}}\right], \quad t_{0}<t<t_{d} \\
V_{t L}\left(t_{d}\right) \cdot e^{-\frac{\left(t-t_{d}\right)}{\tau_{L}}}, \quad t_{d}<t<t_{r}
\end{array}\right.
$$


onde: $R_{t L}$ é a resistência transiente de longa duração, $V_{t L}\left(t_{d}\right)$ é a tensão transiente de longa duração no tempo final de descarga, $\tau_{L}=R_{t L} \cdot C_{t L}$ e $C_{t L}$ é a capacitância transiente de longa duração.

Os parâmetros que modelam a tensão transiente dependem do $S O C$ e são dados por

$$
\left\{\begin{array}{l}
R_{t S}[S O C(t)]=c_{0} \cdot e^{-c 1[S O C(t)]}+c_{2}, \\
C_{t S}[S O C(t)]=d_{0} \cdot e^{-d 1[S O C(t)]}+d_{2}, \\
R_{t L}[S O C(t)]=e_{0} \cdot e^{-e 1[S O C(t)]}+e_{2}, \\
C_{t L}[S O C(t)]=f_{0} \cdot e^{-f 1[S O C(t)]}+f_{2} .
\end{array}\right.
$$

\section{Resultados e Discussões}

Nesta seção são apresentados os resultados obtidos nesta pesquisa e as discussões. Inicialmente, é realizada uma descrição da metodologia adotada para a coleta dos dados e o conjunto de dados obtido nos processos de descarga das baterias. Em seguida, é apresentada a estimação dos parâmetros da parte analítica. Por fim, são apresentados os resultados obtidos a partir da validação.

\subsection{Obtenção dos dados experimentais}

Nesta seção é apresentada a metodologia utilizada para a coleta dos dados experimetais a partir da plataforma de testes. Esta metodologia é dividida em dois momentos. O primeiro momento consiste em conectar a bateria em uma fonte de carregamento utilizando um processo de carga lenta (i.e., $20 \%$ da capacidade nominal) até atingir a tensão de 4,2 V. Este procedimento tem como objetivo garantir que todas as baterias encontrem-se completamente carregadas no início da descarga. No segundo momento as baterias são conectadas à plataforma e submetidas a uma corrente de descarga. Este processo ocorre enquanto as mesmas não atingirem a tensão de cutoff.

Os experimentos foram realizados para perfis de descargas constantes e variados, utilizando oito baterias de Li-Po novas, modelo PL383562-2C. Os dados experimentais obtidos a partir das descarga constantes são divididos em dois conjuntos. O primeiro conjunto é formado por 16 perfis de descargas em $\mathrm{mA}$, que variam de 50 a $800 \mathrm{~mA}$, com intervalos de $50 \mathrm{~mA}$ e os respectivos $T V_{\text {em }}$ (Tempo de Vida experimental médio) em minutos, sendo utilizado para a estimação dos parâmetros do modelo. O segundo conjunto é composto por 15 perfis de descargas em mA, que variam de 75 a $725 \mathrm{~mA}$, com intervalos de $50 \mathrm{~mA}$ e os respectivos $T V_{e m}$ em minutos, sendo utilizado para a validação do modelo, maiores detalhes sobre estes conjuntos de dados pode ser obtido em [4].

Tendo em vista que em um processo de descarga real, as correntes variam de acordo com a operacionalidade do aparelho, o modelo híbrido, foi aplicado também, para correntes de descarga variadas. Estas correntes foram mensuradas a partir das principais funcionalidades executadas em um smartphone, tais como, chamada, envio de sms, acesso a Internet, jogos, música, câmera, visualização de imagens e outras. O terceiro conjunto é formado por 8 perfis de correntes variadas em $\mathrm{mA}$ com os respectivos $T V_{e m}$ em minutos, maiores detalhes sobre este conjunto de dados pode ser obtido em [6]. 


\subsection{Estimação dos Parâmetros do Modelo Híbrido}

Nesta seção é apresentada, brevemente, a metodologia adotada para a estimação dos parâmetros $c$ e $k^{\prime}$ do modelo. A estimação do parâmetro $c$ depende da condição inicial na fonte de carga disponível $y_{1}(0)$ e do valor da capacidade total máxima disponível $y_{0}$, pois $c=y_{1}(0) / y_{0}$. Para determinar $y_{1}(0)$ analisa-se a tendência obtida a partir dos dados experimentais para correntes de descarga muito altas, com isso, $y_{1}(0)=2520 \mathrm{As}$. Para determinar $y_{0}$ considera-se correntes de descarga muito pequenas, então $y_{0}=2821,1 \mathrm{As}$. Portanto, tem-se para o parâmetro $c$ um valor estimado de $c=0,8933$. O parâmetro $k^{\prime}$ é encontrado a partir da substituição de dados experimentais na equação (4). Esta substituição resulta em uma equação transcendental na qual é solucionada com o auxílio do Método de Newton-Raphson. Com isso, determina-se que $k^{\prime}$ é 0,0005 [4]. Os parâmetros referentes à parte elétrica do modelo são obtidos de [5].

\subsection{Validação do Modelo Híbrido}

Nesta seção são apresentados os resultados encontrados na validação do modelo híbrido utilizando correntes de descargas constantes e variadas. Destaca-se que as simulações computacionais foram realizadas na ferramenta computacional MatLab/Simulink.

Na Tabela 1 são comparados, para cada perfil de descarga com correntes constantes, os tempos de vida experimentais médios $\left(T V_{e m}\right)$ com os tempos de vida obtidos a partir das simulações do modelo híbrido $\left(T V_{m h}\right)$ em minutos (min). Também são apresentados os erros provenientes desta comparação, assim como o erro médio do modelo.

Tabela 1: Validação do Modelo Híbrido para Correntes Constantes.

\begin{tabular}{|c|c|c|c|}
\hline Perfil & $T V_{e m}$ & $T V_{m h}$ & Erro $(\%)$ \\
\hline 75 & 606,94 & 636,02 & $4,79 \%$ \\
\hline 125 & 384,76 & 380,02 & $1,23 \%$ \\
\hline 175 & 272,23 & 270,31 & $0,71 \%$ \\
\hline 225 & 203,49 & 209,36 & $2,88 \%$ \\
\hline 275 & 165,17 & 170,59 & $3,28 \%$ \\
\hline 325 & 141,29 & 143,77 & $1,76 \%$ \\
\hline 375 & 123,11 & 124,12 & $0,82 \%$ \\
\hline 425 & 108,38 & 109,11 & $0,67 \%$ \\
\hline 475 & 94,26 & 97,29 & $3,21 \%$ \\
\hline 525 & 86,20 & 87,74 & $1,79 \%$ \\
\hline 575 & 77,84 & 79,86 & $2,60 \%$ \\
\hline 625 & 71,33 & 73,26 & $2,71 \%$ \\
\hline 675 & 65,97 & 67,65 & $2,55 \%$ \\
\hline 725 & 60,69 & 62,83 & $3,53 \%$ \\
\hline 775 & 56,63 & 58,64 & $3,55 \%$ \\
\hline & & & Erro médio $2,41 \%$ \\
\hline
\end{tabular}

Na Tabela 2 são comparados, para cada perfil de descarga com correntes variadas, os tempos de vida experimentais médios $\left(T V_{e m}\right)$ com os tempos de vida obtidos a partir das 
simulações do modelo híbrido $\left(T V_{m h}\right)$ em minutos (min). Também são apresentados os erros provenientes desta comparação, assim como o erro médio.

Tabela 2: Validação do Modelo Híbrido para Correntes Variadas.

\begin{tabular}{|c|c|c|c|}
\hline Perfil & $\left(T V_{\text {em }}\right)(\min )$ & $\left(T V_{m h}\right)(\min )$ & Erro $(\%)$ \\
\hline P1 & 479,67 & 490,35 & $2,23 \%$ \\
\hline P2 & 284,94 & 271,95 & $4,56 \%$ \\
\hline P3 & 322,01 & 333,06 & $3,43 \%$ \\
\hline P4 & 149,38 & 155,06 & $3,80 \%$ \\
\hline P5 & 141,75 & 146,07 & $3,04 \%$ \\
\hline P6 & 126,62 & 124,45 & $1,72 \%$ \\
\hline P7 & 98,51 & 101,21 & $2,73 \%$ \\
\hline P8 & 324,17 & 329,02 & $1,50 \%$ \\
\hline & & & Erro médio $2,87 \%$ \\
\hline
\end{tabular}

A análise dos resultados obtidos a partir da comparação entre os dados experimentais e os calculados pelo modelo para perfis constantes, mostra que o maior erro foi obtido para a corrente de descarga igual a $75 \mathrm{~mA}$, com um valor de $4,79 \%$, e o menor erro durante a descarga de $425 \mathrm{~mA}$, com um valor de $0,67 \%$. Para correntes de descargas variadas é possível observar que o maior erro está relacionado ao perfil 2, com um valor de 4,56\% e o menor erro, relacionado ao perfil 8, com um valor de 1,50\%. Desta análise pode-se concluir que o modelo híbrido apresenta desempenho satisfatório, quando aplicado para correntes de descarga constantes e variadas, tendo erro médio de $2,41 \%$ e 2, $87 \%$, respectivamente.

\section{Conclusões}

Neste trabalho, foi realizada a modelagem matemática do tempo de vida de baterias Li-Po através de um modelo híbrido constituído da união do modelo elétrico para Predizer Runtime e Características V-I de uma bateria com o modelo analítico KiBaM. Este modelo consegue capturar as características elétricas da bateria, assim como os efeitos não lineares que estão presentes no processo de descarga. As simulações com este modelo híbrido foram realizadas na ferramenta computacional MatLab/Simulink, tanto para descargas com correntes constantes, como variadas. Os resultados das simulações foram comparados com uma série de dados experimentais, obtidos a partir de uma plataforma de testes, desenvolvida pelo Grupo de Automação Industrial e Controle (GAIC) da Unijuí. Os testes experimentais foram realizados com baterias do tipo Li-Po, modelo PL383565-2C.

A literatura afirma que modelos são considerados acurados para a predição do tempo de vida de baterias se apresentarem um erro médio inferior a 5\%. Das análises realizadas conclui-se que o modelo híbrido é satisfatório no que se refere à predição do tempo de vida de baterias de dispositivos móveis, pois para descargas com correntes constantes o modelo apresentou um erro médio de $2,41 \%$, e nas descargas com correntes variadas este valor foi de $2,87 \%$ [9]. 


\section{Agradecimentos}

Os autores agradecem à Unijuí e a CAPES pelo apoio financeiro e ao GAIC, pela infra-estrutura.

\section{Referências}

[1] M. Chen and G. Rincón-Mora. Accurate electrical battery model capable of predicting runtime and i-v performance. IEEE Transactions on Energy Conversion, 21(2):504511, June 2006.

[2] C. Chiasserini and R. Rao. Pulsed battery discharge in communication devices. In Proceedings of the 5th Annual ACM/IEEE International Conference on Mobile Computing and Networking, MobiCom'99, pages 88-95, USA, August 1999. ACM.

[3] M. Doyle, T. F. Fuller, and J. Newman. Modeling of galvanostatic charge and discharge of the lithium, polymer, insertion cell. Journal of the Electrochemical Society, 140(6):1526-1533, 1993.

[4] L. Fransozi. Modelagem matemática do tempo de vida de baterias de lítio Íon polímero através de modelos híbridos. Dissertação de mestrado, Universidade Regional do Noroeste do Estado do Rio Grande do Sul, 2015.

[5] T. Kim. A hybrid battery model capable of capturing dynamic circuit characteristics an nonlinear capacity effects. IEEE Transactions on Energy Conversion, 26(4), December 2011.

[6] R. S. Kusiak. Modelagem matemática do tempo de vida de baterias de lítio Íon polímero a partir de modelos híbridos considerando correntes de descarga variáveis. Dissertação de mestrado, Universidade Regional do Noroeste do Estado do Rio Grande do Sul, 2016.

[7] J. F. Manwell and J. G. McGowan. Lead acid battery storage model for hybrid energy systems. Solar Energy, 50(5):399-405, 1993.

[8] D. Rakhmatov and S. Vrudhula. An analytical high-level battery model for use in energy management of portable electronic systems. In Proceedings of the 2001 IEEE/ACM International Conference on Computer-aided Design, ICCAD '01, pages 488-493, Piscataway, USA, 2001. IEEE Press.

[9] M. F. Triola. Introdução à Estatística. LTC, Rio de Janeiro - RJ, 1999.

[10] J. Zhang, S. Ci, H. Sharif, and M. Alahmad. An enhanced circuit-based model for single-cell battery. Applied Power Electronics Conference and Exposition, 2010. 